\title{
HYDRAULIQUE
}

\section{Quelques considérations sur le prix de revient de l'énergie hydraulique et sur le programme d'électrification en France}

\author{
par M. Massonneau, Inqénieur à la Compagne des Chemms de fer du Midi
}

Les statistıques officielles publiées annuellement montrent qu'au point de vue produclion du charbon, la France est largement déficitarre el qu'll luı faut importer de l'Elranger, au cours du dollat, les tonnes de charbon nécessarres à ses industries de toute nature (1). On est alors élonné, dans certams milıux non initiés, de vou que le Gouvernement ne poursuit pas p'us act1vement la politique, instaurée en 1919, d'aménagement de toutes les chutes d'eau de notre terrilore On pense qu'avec les millons de $k w-h$ hydrauliques produits, la France serait dispensée d'acheter au dehors le combustible manquant De là, à voir une répercussion dirccle sur la vie chère, il n'y a qu'un pas

On se demande aussi, quelquefors, comment il se fait que la mise en service d'usines hydrauhques puissantes ne fasse pas baisser le lonnage du charbon importé (2). C'est, en effet, ce qui arriverall si les demandes de l'industrie restaienl stalionnarres, mais on sal qu'll n'en est rien au fur et à mesure que se déveIoppe le machmisme, les besouns croussent de parr et, ansı, malgré l'augmentation possible de l'extraction houllère, les demandes en énergie ne cesseront de croître Cependant il résulte de documents officiels qu'en Sursse, où le développement de l'industrie hydro-élechique a élé très intense ces dermères années, la consommation en charbon a dimınué de 600000 tonnes.

Mais, s'il est vrai que l'eau ulılısée dans les Lurbınes des usınes hydro-électriques ne coûte rien, à l'inverse du charbon que consomment les usmes thermuques, il y a un élément dans l'aménagement des chutes d'eau qu coûte fort cher, ce sont les travaux que nécessile cel aménagement barrage. canaux d'amenée, conduites sous pression, bâtıment d'usınes et canal de fuite

De plus, quelle que soit la siluation de l'usine, plaine ou montagne, l'exécutıon doit se poursurvre sur plusieurs années, 3 ou 4 ans pour une basse chute, 5 ou 6 pour des travaux de montagne silués au-dessus de la cote 1200 , là où les campagnes de travaux ne durent que $\delta$, et même moins de 6 mols Au contraire, une puissanle usıne thermıque ne demande, en général, pas plus de 30 mois pour son édificalıon.

Le capilal nécessare pour solder la dépense des travaux de l'usine hydro-électrique ayant dû ètre constılué, dans le cas le plus général, avant tout commencement des travaux, il en résulte que les intérêts intercalaıres vont jouer pendant toute la durée de l'exécution, c'est-à-dıre avant même qu'un kw.-h ne solt produit par l'usme. Or, on compte qu'aux taux actuels de l'argent, et pour des travaux durant 6 ans, le capital de premier établissement aura à supporler une majoration qui pourra atteindre $50 \%$. Les charges de ce capital supplémentare dimnueront donc d'autanl le revenu de l'usine, lorsqu'elle sera en plein rendement

(1) En 1925, ce déficit était d'environ 23 millions de tonnes

(2) En 1925, les usines en service produsaient 3 milliards et demi de kw.-h., remplaçant 3 millions et demi de tonnes de charbon. importé.
De plus, il esl bien rare que l'énergie hydro-électrıque solt ulılısée sur le lieu même de sa production, il y a donc heu de compter, en plus, les postes élévateurs et abaisseurs de tension el une ligne de transport à haute iension, souvent fort longue et qui, pour des raisons de sécurité, doit être double.

Par contre, l'usme thermique sera construite, sauf dans des cas très particuliers utılisation sur le carreau des mines des poussières de charbon, sur le lieu même de distribution de l'énergie.

Par alleurs, une usine établie sans bassin d'accumulation, une usine au fil de l'eau comme on l'appelle, voit sa production d'énergie commandée par le débit de la rivière. Forte pendant les pérıodes de hautes eaux, elle diminue considérablement pendant les périodes d'étıage.

De plus, si la consommation de sa production n'est assurée que pour une partıe de la journée, ll y a perte d'énergie pendant toule la durée de l'arrêt des machines, l'eau qui arrive à la prise ne pouvant être retenue.

Le prix de revient du kw.-h. produit dans une telle usine peut rarement, à moins de circonstances locales très favorables, souLenir la comparason avec celur d'une usine thermique.

L'utılısation des installatıons peut, heureusement, s'améliorer beaucoup par certains aménagements spéciaux. solt par l'exécution d'un bassin d'accumulation quand la chose est économlquement possıble, soit par connexion avec une usine située dans une régıon à régıme hydraulıque différent, soit même par les deux procédés ensemble

El d'abord que faut-il entendre par prix de revient du kw.-h. ?

La Curculaire ministérieile du $1^{\mathrm{er}}$ août 1921 va nous permettre de répondre à cette question c'est le rapport existant entre " le montant annuel des charges de toutes natures que l'Entreprise a à couvrir (dépenses d'établissement, charges obligataires, rémunération du capital-actıons, dépenses d'entretien et de renouvellement, dépenses d'exploitation) " et "la production totale d'énergie qui peut ètre obtenue, compte tenu du coefficient d'ulthsalion que cetle énergie comporte, en raison de sa régularité el de sa constance ".

Si le numérateur de ce rapport est suffisamment défin, le dénominateur est loin de l'ètre.

En effet, prenons le cas d'une usine au fil de l'eau. Sa puissance mensuelle qui, en supposant la hauteur de chute constante (1), est fonction du débit de la rivière sera par conséquent très variable.

Si l'on considère les débits moyens mensuels de la rivière, Lorrent des Pyrénées par exemple, on peut tracer le graphique des puissances de l'usine qui a l'allure ci-contre (fig.1).

On remarque sur ce graphique que cette puissance comprend deux parties : l'une continue pendant toute l'année, celle située

(1) Ce n'est vraiment le cas que pour les hautes chutes. 
au-dessous de l'horizontale $A B$, l'autre variable suivant le mois considéré, située au-dessus de $A B$.

La puissance représentée par la tranche inférieure a la valeur maximum, par contre, celle que représentent les rectangles supérieurs a une valeur beaucoup mondre, quelquefors même n'en a aucune, la plupart des clients s'accommodant mal d'une livraison discontinue d'énergie.

De plus, ll est rare 'qu'une telle entreprise pusse placer, pendant 'es premières années d'exploitation, toule l'énerg e produite, même celle continue.

Dans ces condit ons, on se rend compte combien le calcul du prix de revient de l'énergie hydraulıque comporte d'aléas. On peut même dire que ce prix varie du simple au double et même plus ; mais, toutecois, qu'il dort diminuer quand la consommation augmente.

Dans un rapport au III Congrès de la Houille Blanche, M C. Duval a indiqué que pour une utilisation passant de 2.000 à $4.500 \mathrm{~h}$. le prix de revient du kw. h. était réduit de moitié.

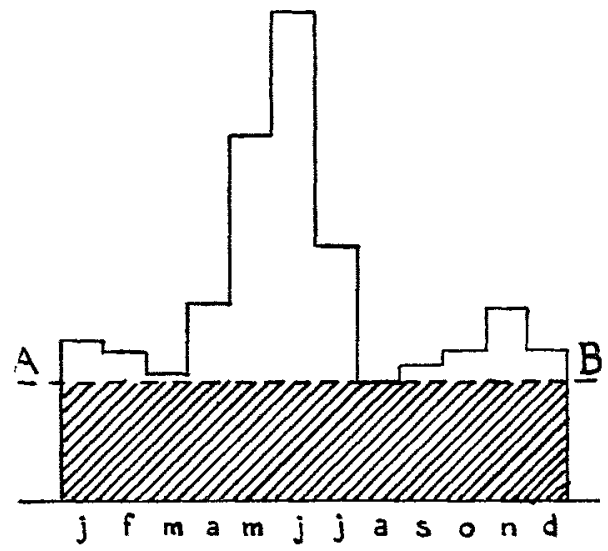

Fig. 1.

Tout l'effort de l'industriel doit donc tendre vers une uthlisation maximum des installations de son usine.

Un autre élément qui joue dans le calcul du prix de revient de l'énergie hydraulıque est le suivant : l'usine doıt être construite immédiatement pour la production maximum; on ne peut, en effet, revenir ultérieurement, pendant l'exploitation, pour augmenter les dimensions de la prise d'eau ou les dimensions du canal d'amenée. Tout au plus peut-on procéder seulement à l'établissement d'une partie des conduites sous pression et réserver l'installation d'un ou de deux groupes turbo-alternateurs dans le bâtiment d'usine. On est donc contraint, comme conséquence, d'engager, de suite, la presque totalité des dépenses de premier établissement.

On voit par cet exposé combien il est diffıcile d'établir exactement le prix de revient d'une installation hydro-électrique. A ces difficultés s'en ajoutent d'autres : les aménagements sont en général peu comparables, tant au point de vue des ouvrages proprement dits que de leur situation géographique, des ressources locales, des sujétions d'exécution, etc... Depuls la guerre un autre facteur, l'instabilité des prix, fait qu'il est bien difficile de comparer deux installations établıes à des époques différentes. De plus, la plupart des industriels répugnent à faire connaître le montant de leurs dépenses d'établissement.

En dehors des installations d'avant-guerre, pour lesquelles, comme nous le verrons par quelques exemples, les prix sont déjà très différents, nous donnerons quelques prix concernant des usines suisses où, comme on sait, la valeur de la monnaie a peu varié. Nous nous sommes servis pour ces calculs, d'une part, d Guide publié, en 1926, par l'association suisse pour l'aménage ment des eaux dans ce pays et, d'autre part, des indications don nées par M. Arbelol dans son rapport présenté à la Conférene mondıale de l'énergie, à Bâle, en 1926 .

L'établıssement du prix de revıent du kw.-h. dans une usin thermique présente beaucoup moins d'aléas : on admet que, pou une même purssance, le coût des travaux est environ trois $\hat{1}_{0}^{\prime}$ moindre Les charges fixes sont donc moindres; mais, par contr les charges proportionnelles au nombre de kw.-h. produits son bien supérieures et cela en raison de la consommation du charbo matière quı coûte cher au regard de l'eau qui ne coûte rien.

\section{USINES AU FIL DE L'EAU, SANS RÉSERVOIR JOURNALIER.}

Quand, avant 1914, une usine hydro-électrique était établi pour des besorns bien définis - électro-chimie, électro-métallu gie, et consommait son énergie sur place, le prix de revient d kw.-h. était, en général, toujours très avantageux.

En effet, l'usine établie dans la partie la plus pentée du cou d'eau et équipée pour le débit moyen, produisait le $\mathrm{kw}$ - $\mathrm{h}$, a prix minimum. C'était l'âge d'or de la houlle blanche. La I de 1919, dans un but d'intérêt général, ne permet plus de te aménagements dont la possıbilité est, d'ailleurs, devenue de pl en plus rare.

\section{USINES AVEC RÉSERVOIR JOURNALIER.}

Une usine hydro-électrique est souvent créée dans le but fournir du courant pour la lumière ou pour la traction, quelqu fois pour ces deux raisons ensemble.

Dans ces cas, ll est nécessaire que, durant certaines heures la journée, la puissance produite sort augmentée, doublée mêm l'armement de l'usine étant prévu en conséquence. C'est ce qu' appelle satisfaire aux pointes : pour la lumière le moment. produit à la tombée de la nuit, pour la traction au moment le trafıc devient le plus intense Pour permettre de passer pointes, il faut dısposer d'une quantité d'eau mise en réserve moment de la demande minimum, la nuit par conséquent. A effet on établit, en tête de la prise d'eau, un bassin d'accumu. tıon journalière. Mars ces réservoirs sont, en général, d'un é blissement onéreux et viennent ainsi grever les dépenses de p mier établissement et, par voie de conséquence, le prix du kw. Il y a, dans chaque cas d'espèce, une étude financière à faire po savoir sı l'opération se présente ou non dans des conditions ava tageuses.

Parmi les installations de ce genre, en basse chute, on pe citer :

$1^{\circ}$ En France. - Les usines établies sur la Durance, celles Jonage, de Chancy-Pougny, etc...

L'usine de Jonage, une des plus anciennes de France, a puissance installée de $20.000 \mathrm{CV}$ (11 mètres de chute). Les d penses se sont élevées à 36.000 .000 francs, ce qui donne un $p$ de revient du kw.-h. de 20 centimes environ (cher).

L'usine de Chancy-Pougny (1920-25) chute nette $8 \mathrm{~m}$. $6 \mathrm{~m}$. 30, débit maximum 450 mc.-s., moyenne 260 me.-s., co porte une puissance installée de $47.500 \mathrm{CV}$ el fournit son éner à la Société " Energıe Electrique du Rhône et Jura ». La produ tion annuelle possible est de $150.000 .000 \mathrm{kw} . \mathrm{h}$., celle con tante de 65.000.000.

Le prix de revient du kw.-h. conslant a été de 24 centim (8 c. suisses). 
$2^{\circ}$ En Suisse. - L'usine d'Eglisau (1915-1920) chute 10 m. 90$8 \mathrm{~m}$. 70, débit de 150 à $380 \mathrm{mc}$.-s., comporte une purssance installée de $42.000 \mathrm{CV}$. La production annuelle possible est de $200.000 .000 \mathrm{kw}$. -h., celle constante de 88.000 .000 . Le prix de revient du kw.-h. constant est de 8 c. 4 suisses.

Parmi les hautes chutes, l'usine de Kueblis (1919) chute 330 mètres, réserve $90.000 \mathrm{~m}^{3}$, débit utilisé $10 \mathrm{mc}$.-s. comporte $35.000 \mathrm{CV}$ installés. Elle fournit son énergie à des chemins de fer de montagne, au réseau des F.M., du N.E. et à la ville de Zurıch.

La production annuelle possible est de $135.000000 \mathrm{kw}-\mathrm{h}$, celle constante de 105 millions.

Prix de revient du kw.-h. constant, 5 c 8 suisses.

La conception d'une usıne hydro-électrique isolée, même accolée à un réservoir journalier est, maintenant, une erreur qu'il ne faut plus commettre; on arrive presque toujours, en raison du coût du réservoir, à un prix de revient de l'énergie plus élevé que celur produit par l'usine thermique.

\section{USINES HYDRAULIQUES ET THERMIQUES CONNECTÉES} ENTRE ELLES.

A l'heure actuelle, à moins de crrconstances particulières (cas des usines d'électro-chimie ou d'électro-métallurgie) la tendance consiste à établrr la liasson des usines hydrauliques entre elles et leur réunion aux gros centres de consommation. La connexion des centrales entre elles est opérée au moyen de lignes de transport à haute tension. Les usines étant établies sur des rivières ou torrents à régimes différents, pourront, à certaines époques de l'année, se porter secours. Cet ensemble peut même avantageusement, être complété par des usines thermiques construites sur le carreau des mines et utılisant les déchets de charbon, ces dernières n'étant mises en marche chaque année que pendant les mois de creux du groupement.

On peut même prévoir une connexion plus étendue, par exemple avec des usines thermiques étables sur le lieu de consommation et dont le régime de marche serait légèrement modifié au profit de la régularisation de l'ensemble. Un exemple de cet appuı mutuel est fourni par la réunion de l'usine d'Eguzon de la Compagnie de Paris-Orléans avec la Centrale de Gennevilliers, près de Paris, de l'Union d'Electrıcıté, pour servir d'une part à l'électrification du réseau Paris-Orléans, et, d'autre part, fournir en hautes eaux un appoint aux usines thermiques de la régıon parisienne.

Sans cet appu l'usine d'Eguzon aurait une mauvaise utilsation de ses $50.000 \mathrm{kw}$. installés, le débit pouvant en effet tomber de $88 \mathrm{~m}^{3}$ en hiver à $1 \mathrm{~m}^{3}$ en été.

Le prix moyen du kw.-h., escompté au moment de la construction de l'usine, était de $10 \mathrm{c}$. étant entendu que, par le jeu des connexions ci-dessus, toute la production serait utulisée S'il n'en étalt pas ainsi, la discrimination entre énergie permanente et énergie de hautes eaux conduirait à augmenter dans une proportion notable le chiffre ci-dessus et ce, en raison de la moindre valeur de l'énergie des hautes eaux.

\section{USINES HYDRO-ÉLECTRIQUES AVEC RÉSERVOIR ANNUEL.}

Une telle usine est, en général, une usine de haute chute. Le réservoir est souvent un lac de haute montagne qui emmagasine les eaux de fonte des neiges. L'usine peul être exécutée avec canal en charge ou à écoulement libre. Dans le premier cas, le débít dans le canal d'amenée dépend uniquement de l'appel de l'usine. Une telle usine est utilisée soit pour faire les pointes, soit pour fournir de l'énergie prise sur la réserve au moment où il y a pénurie d'eau dans le cours d'eau. De plus la conjugaison d'une telle usine avec d'autres établies sans réserves saisonnières permet de régulariser l'énergie de hautes' eaux de ces usines et par suite de valoriser cette énergie qui, sans cela, aurait peu de valeur.

Dans le second cas, l'usine peut être utilisée comme celle précédente mais les manœuvres fréquentes à la prise d'eau que cette utulisation comporte entraînerarent des pertes d'eau souvent importantes à cause de l'impossibilité du réglage du débit des robinets à la demande.

En France, les usines d'Orlu, des Sept-Laux sont du premier type, celle d'Eget est du second.

L'usine d'Eget, commencée avant la guerre et terminée en 1923 , est alimentée par le réservorr de l'Oule de $6.500 .000 \mathrm{~m}^{3}$. Elle comprend $35000 \mathrm{CV}$ installés. Sa production annuelle permanente est de $85.000 .000 \mathrm{kw}$.-h., dont le prix de revient a été de 5 c. environ.

En Suisse, on peut citer :

10 L'usine de Barberine (1919-1925) chute nette 735 mètres, réservorr de $39.000000 \mathrm{~m}^{3}$, puissance prévue $62.400 \mathrm{CV}$, puissance installée $46.800 \mathrm{CV}$, production annuelle constante 60.000 .000 kw.-h.

\section{Prix de revient du kw.-h. : 11 c. 2 suisses.}

$2^{\circ}$ L'usine de Heidsee (1917-1920) chute nette 592 mètres, réservoir de $810.000 \mathrm{~m}^{3}$, puissance installée $13000 \mathrm{CV}$, production annuelle constante $14.000 .000 \mathrm{kw} .-\mathrm{h}$., prix de revient du kw.-h, 8 c. 4 suisses.

$3^{\circ}$ L'usine de l'Etzel (en projet) chute nette 470 mètres, réservoir de $96.500000 \mathrm{~m}^{3}$, puissance prévue $120.000 \mathrm{CV}$, production annuelle totale et constante $125.000000 \mathrm{kw}$.h., prix de revient escompté du kw.-h. 8 c. 5 suisses.

\section{USINES HYDRO-ÉLECTRIQUES AVEC RÉSERVOIRS ANNUELS ALIMENTÉS EN PARTIE ARTIFICIELLEMENT.}

On peut même, dans certains cas, concevoir une alimentation supplémentaire, artificielle, du réservoir annuel au moyen du pompage des hautes eaux. Celles-ci, qui passent en effet par dessus les déversoirs des usines sans aucun profit pour personne, peuvent être refoulées au moyen des conduites forcées et du canal d'amenée jusque dans le bassin supérieur.

C'est ainsi que la Société d'électro-chimie, d'électro-métallurgie et des Aciéries d'Ugine a installé dans son usine de Belleville une batterie de pompes destinées à refouler dans le lac de la Grrotte les eaux en excédent aux heures ou aux saisons où l'on dispose à la fois de force et d'eau au delà des besoins.

Les eaux mises en réserve dans le lac de la Girotte sont d'ailleurs utilisées directement dans la même usine de Belleville à la mise en jeu de groupes hydro-électriques. Le courant destiné au pompage provient du réseau de la Société auquel l'usine de Belleville est reliée en parallèle. L'eau à pomper est prélevée directement sur le Dorinet, torrent qui passe devant l'usine.

La prise, très simple, comporte un barrage, une passe à gravier, une grille de défeuillage, un dessableur double avec grilles de bois stabilisatrices et un bassin de décantation double.

La station comprend deux groupes : sur le même axe sont montés un alternateur, une turbine et une pompe; le tout constituant un groupe. Nous n'avons aucune indication sur le prix du kw.-h. produit.

Cette station donne, d'ailleurs, toute satisfaction. 
A une échelle plus modeste, la Compagnie du Midi a commencé les travaux d'installation d'une station de pompage au lac d'Artouste. Les pompes refouleront dans le lac aménagé en réservorr des eaux qui, sans cette installation, se jetteraient dans l'ancien émissaire du lac et ne pourraient travaller que dans les usines inférieures.

USINES HYDRO-ÉLECTRIQUES EN ECHELON, ALINENTEES PAR IN RÉSERVOIR ANNUEL.

Le programme peut comporter l'établissement de plusieurs usines échelonnées en cascade, sur un mème cours d'eau

Dans cet ordres d'idées, et pour l'électrification de son réseau, la Compagnie des Chemins de fer du Midı a aménagé, dans la vallée d'Ossau (Basses-Pyrénées) un groupe de trois usines en échelon : Artouste, Miegebat el Hourat, la première devant servir, au moyen des_réserves accumulées dans le lac d'Arlouste tion dont la valeur peut atteindre le double de leur puissanc; moyenne. En conséquence, elles ont été armées largement ef dotées de réserves journalì̀res importantes $\left(200000 \mathrm{~m}^{3}\right)$ éta. blıes partıe dans le canal même, partıe à cıel ouvert.

Bien que l'aménagement d'ensemble des usines de l'Ossa ne sout pas encore complètement achevé, les travaux sont suff. samment avancés pour permettre de chiffer, avec assez d'exal tıtude, le prix de revient maximum du kw.-h.

La production moyenne annuelle continue de cet ensemble élant de 240 millions de $\mathrm{kw}$. $-\mathrm{h}$., ll semble que ce prix doive reste inférıeur à $12 \mathrm{c}$.

Par ailleurs, la Compagnıe du Mıdı possède déjà un ımportant réseau de lignes de transport à haute tension 60.000 el 150.00: volls relianl ses uslnes de Soulom, Egel el de l'Ossau, à Bor deaux d'une part, el Portel-Sant-Simon d'autre part. Ce réseat sera bientôt prolongé par une nouvelle artère à 150.000 volt

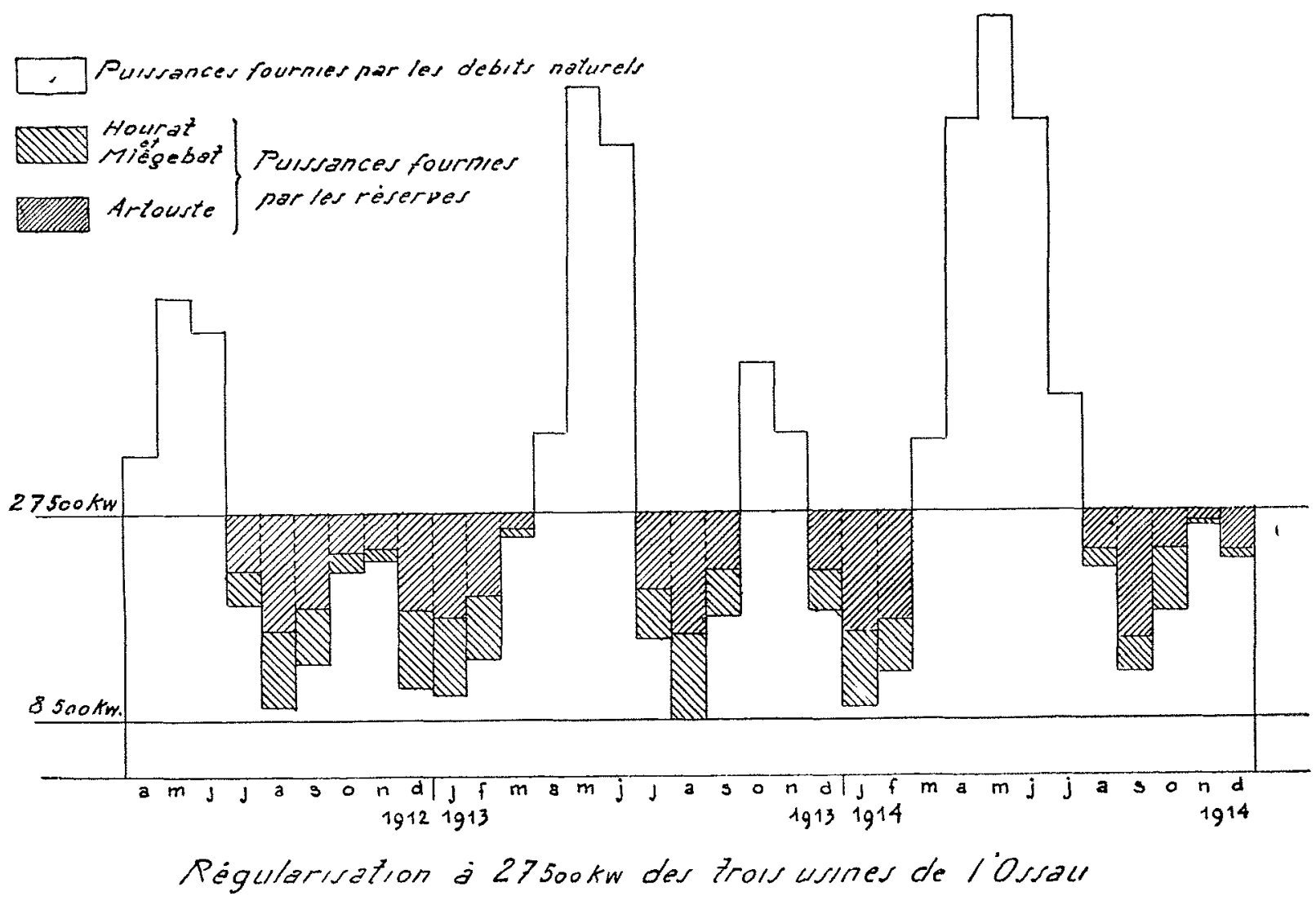

Fig. 2.

d'une capacité de $23.000000 \mathrm{~m}^{3}$, à la régularisation complète des deux autres usines situées en aval

Ce fait est nettement représenté sur le graphique (fig 2) où sont figurées les puissances des usines de la vallée d'Ossau pour une période de trois années (1912 à 1914) La partıe inférieure (rertangle en blanc) indique la puissance au fil de l'eau des deux usines basses, on volt que, dans ce cas, la tranche de puissance continue serait celle correspondant au mois d'août 1913, c'est-à-dıre $8500 \mathrm{kw}$.

En outre, sur ce même graphique, on a représenté, d'une part par des rectangles à hachures serrées, les puissances fournıes par l'usine d'Artouste fonctionnant avec les eaux de son réservoir annuel et, d'autre part, par des rectangles à hachures lâches les puissances fournies par cette même eau dans les deux usines inférieures. On remarquera que, par le jeu du zéservoir, la lranche de puissance continue passe de 8500 à $27.500 \mathrm{kw}$.

De plus, les usines du Hourat et de Miegebat, exécutées avec canaux en charge, doivent pouvoir assurer des pointes de trac-
Portet-Saint-Simon-Usine du Pinet, sur le Tain, appartenali à la Société "Le Rouergue ", réalisant ansi la conjugaison de usines des Pyrénées avec celles du Massif Central, conjugaiso extrêmement intéressante étanl donné que les régımes des coll d'eau sont différents, et, qu'en général, la période des haul eaux de l'un correspond à la pérıde des basses caux de l'aulr

Dans ces condilions, on peut prévorr que la connexion ent les différents réseaux cités plus haut permettra des échang d'énergıe aux différentes époques de l'année, et qu'aunsi toll l'énergie produite par les usines de l'Ossau sera utılisée. On pi' voit que, de ce fait, le prix de revient du kw. $\mathrm{h}$. pourra ôt encore abaissé.

En rapprochant les différents prix ci-dessus de ceux indiq̣u par M. Arbelot dans le rapport cité plus haut pour le prix $d$ revient du $\mathrm{kw},-\mathrm{h}$. thermique (charbon à 120 francs) aux bart de l'usine et en fonction du nombre d'heures d'utilisation ? rappelés ci-dessous :

Pour 2.000 heures........... $26 \mathrm{c}$. 
Pour 4.000 heures.. . . ....... 19 c.

Pour 6.000 heures............. $16 \mathrm{c}$.

On voil que, malgré les diffıcultés et les imprécisions auxquelles on se heurte dans une élude des prix de revient comparés de l'énergie hydraulique et thermique el dans certaines conditions d'uthlisation des installations, les prix de revient de ces deux énergies doivent tendre l'un vers l'autre.

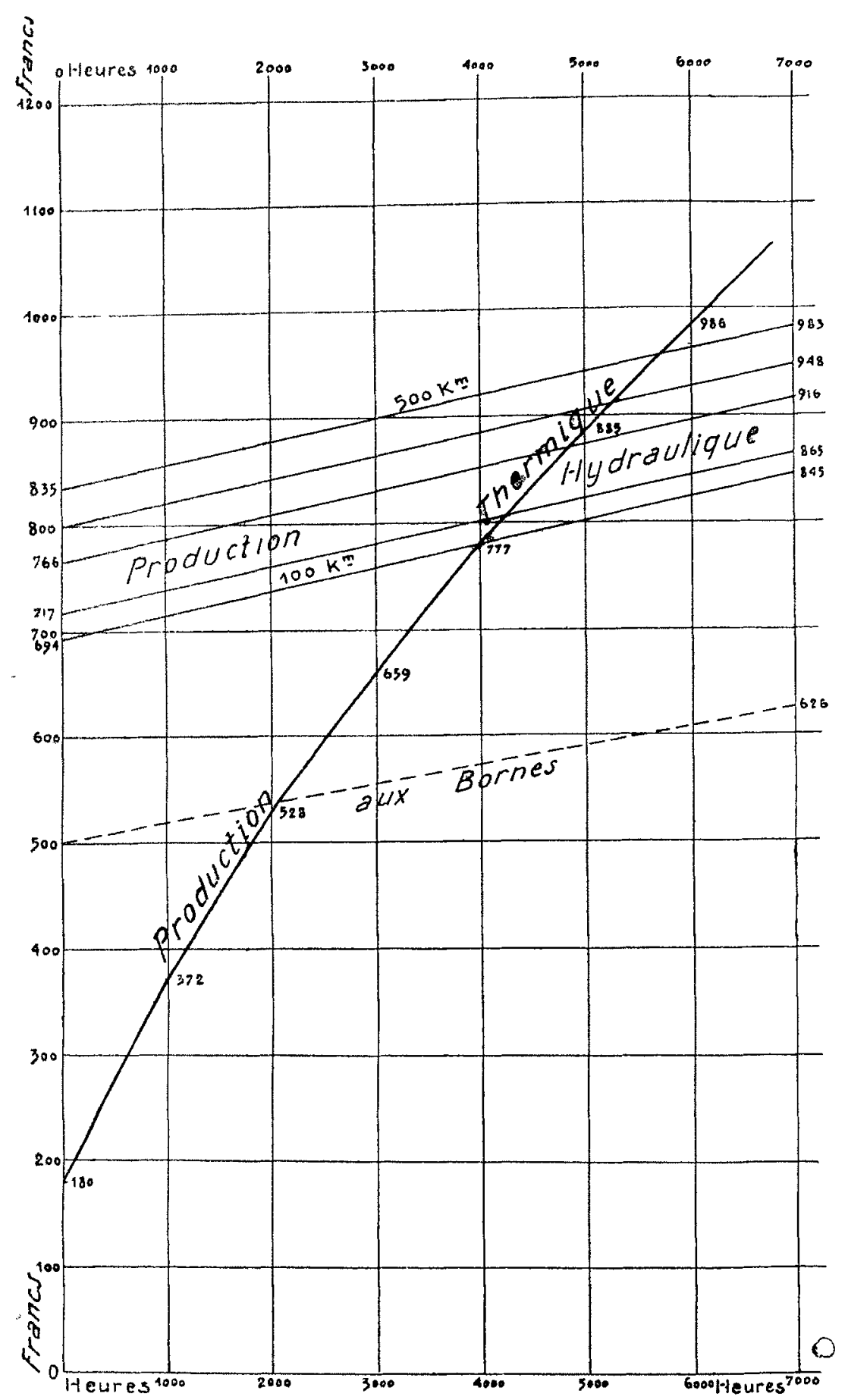

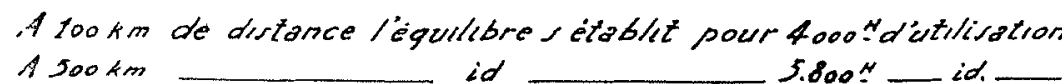

Fig. 3. - Graphique des prix de revient d'un k.w.

M. Arbelot a montré que cel équilıbre étart attennt pour une utilisation de 4.000 à 5800 heures, suivant que la chute est à 100 ou à $500 \mathrm{~km}$ du point d'utilisation. Dans le cas d'une consommation sur place cet équilibre a lieu aux environs de 2.000 heures (fig. 3).

\section{Le programme d'électrification en France.}

Bien que la France soit le berceau de la houille blanche, les grandes installations de forces hydrouliques étaient, avant la guerre, peu nombreuses. Il a fallu la grande épreuve de 19141918 et la pénurie de charbon qu'elle engendra pour amener une amélioration générale des entreprises hydrauliques.

C'est en effet vers la fin de l'année 1918 que le Gouvernement chargea le Conseil supérieur des Travaux publics d'étudier un programme général d'intensification de notre outlllage national.

En ce qui concerne les questions des forces hydraulıques, il s'agissarl de déterminer les besoins probables et le plan d'exécutıon des usines et des lignes dont $1 \mathrm{l}$ y avait lieu de poursurvre la réalisation pendant une période de 10 ou 15 années.

Un premier comité fut créé qui s'occupa des ressources et de l'aménagement des forces hydraulıques; il effectua, pour la première fors, un recensement des usines de quelque importance, en service, en construction et en projet. Il porta ensuite son effort sur l'amélioration souhartable du régime administratif des chutes, il préconısa la constitution systématique de réservoirs d'accumulation, indiqua le mécanisme de l'aide financière pour assurer leur exécution, apportant ainsi une importante contribution aux travaux préparatorres de la loi de 1919

Le deuxième comité, qui devait s'occuper du plan général des distrıbutions d'énergie électrique et des moyens de satisfaire à leur développement, avait une tàche bien plus ardue : il devait, d'une part, évaluer les ressources existantes, et, d'autre part, chiffrer les besoins futurs pour une période de 10 à 15 ans et aviser aux moyens d'y satisfaire.

Un troisième comité était chargé, d'une part, de rechercher les règles générales d'unification en matière d'électrification des chemins de fer, et d'autre part, d'établir les tableaux des lignes à électrifier sur les différents réseaux, avec leur classement par ordre d'urgence.

Enfin, un comité spécial devait étudier l'aménagement du Rhône et de la Dordogne.

La loi du 16 octobre 1919, promulguée à son heure, est devenue suivant la voie tracée par les différents comités, la charte de constitution des usines Elle supprima totalement le commerce si néfaste de certains spéculateurs connus sous le nom de "barreurs de chutes". De plus, elle permit l'éclosion, en nombre considérable, de projets d'installations hydro-électriques, dont l'exécution fut malheureusement entravée par des conditions économiques défavorables.

Dans son rapport déjà cité, M. Arbelot montre que, pour satisfaire à un accroissement de vente dans une année moyenne de 500 millions de kw.-h., qui correspond aux besoins normaux de l'industrie, il faut en produire environ 750 millions (75 à $95.000 \mathrm{kw}$.). Une pareille quantité d'énergie ne peut être demandée entièrement à des installations hydro-électriques. Il faut, comme nous l'avons montré, qu'elles solent économiquement réalisables, c'est-à-dire que l'énergie par elles produite soit susceptıble d'une utilisation de 4.000 à 5.800 heures suivant la position du lieu de consommation (fig. 3). Il y aura done toujours nécessité de construire des usines thermiques et e'est par leur connexion avec les grandes Centrales hydrauliques que pourra être réalisé, d'une manière rationnelle, le grand programme d'électrification de notre pays.

Si nous jetons un coup d'œil en arrière, nous constatons, ainsi 
que nous l'avons dit plus haut, que les aménagements de houlle blanche étaient en France, avant guerre, peu développées.

En 1903, dans les Alpes du Nord, qui ont vu éclore l'ndustrie hydro-électrique, on comptait seulement $107.000 \mathrm{kw}$. de puissance installée; en 1919, ce chiffre dépassatt 516.000 .

Dans les Pyrénées, on comptait $80.000 \mathrm{kw}$. avant 1914. Ce chiffre est passé à plus de 200.000 et les projets en cours vont le porter au delà de $300.000 \mathrm{kw}$. Le progrès a été plus grand encore dans le Massif Central - la puissance moyenne, qui étart de $40.000 \mathrm{kw}$. avant la guerre, est passée à $160000 \mathrm{kw}$., solt une augmentation de $300 \%$. Dans le Jura, dans le bassin de la Garonne, en Normandie et en Bretagne, on a installé des chutes. Les renseignements que nous possédons permettent d'évaluer l'ensemble de la puissance mstallée des usmes françasses de houille blanche à plus de 1 million $1 / 2$ de $\mathrm{kw}$.

$\mathrm{Si}$ on ajoute à ce chiffe les puissances que représentent les usines qui ont fart l'objet d'une demande en concession et celles en projet, on peut prévorr, sans crante d'êlre trop optımiste, que c'est une puissance installée de $3.000 .000 \mathrm{kw}$. qui sera atteinte dans le délal d'une dizaine d'années.

Mars, comme nous l'avons déjà dit précédemment, ı̣l ne s'agit pas seulement de produire de l'énergie électrıque, ll faut en plus, dans la plupart des cas, pouvorr la transporter jusqu'aux centres de consommation.

L'industrie privée peut continuer, comme elle l'a fait jusqu icl, à investir ses capitaux dans l'établissement des usines, quitte à farre appel au régime d'aide financière instıtué par les lors du 16 octobre 1919 et 29 juillet 1922. Mais en ce qui concerne le transport de l'énergie électrique, c'est à l'Etat qu'il appartient de créer les grands réseaux haute tension, comme ll l'a fart, d'alleurs, pour les vores ferrées des grandes Compagnies En effet, dans un cas comme dans l'autre, il s'agit d'engager d'énormes capitaux en vue d'un intérêt général.

De plus, nous avons vu que la création de grands réservorrs étart nécessaire pour régulariser la production des usines hydroélectriques et que leur établissement coûtart fort cher. Là encore où l'nntérêt général est en jeu, il convient que l'Etat en prenne l'exécution à sa charge. Nous avons en effet montré que l'eau accumulée et déversée dans les moments de grande sécheresse étart d'un secours puissant pour les usines établies au-dessous des réservorrs; mais ce n'est pas son seul effet : cette eau permet, de plus, l'rrrigation des terrains situés sur tout le parcours du cours d'eau apportant ansi la richesse à tonte une région.

\section{LEs Forces hydrauliques EN EUROPE.}

A cette heure, tous les Etats de l'Europe ont plus ou moins activement entrepris l'aménagement des ressources hydrauli. ques de leur sol national.

Dans certans pays, comme la Suisse et l'Italie, où le sous-sol ne renferme aucun combustible, mais où, par contre, les rivières et les lacs de montagne sont abondants, on devat tout naturellement volr éclore une politique franchemenl tournée vers l'uti. lisation de la force hydraulıque. En fait, ll est rare de trouver, en Suisse, un petıt village non éclarré par l'électricité.

D'après une statıstique offıcielle, il y avait, en Italie, fin 1922, une pussance hydraulique installée de $2.000000 \mathrm{kw}$.

En Suisse, les installations hydro-électriques ont passé, depuis la guerre jusqu'en 1925 , de 40.0000 à $1380.000 \mathrm{kw}$; en outre $200.000 \mathrm{kw}$. étaient en construction fin 1925.

L'Espagne et le Poriugal possèdent aussi, surlout la premère, d'immenses réserves de puissance en houille blanche; mais, jusqu'ıcı, bien que le combustible à bon marché soit peu abon dant, l'utllisation de l'eau pour produrre de l'énergie y est encore peu développée.

C'est en Norvège, à Rjukanfos, que se trouve la plus gigan tesque des installations hydro-électriques de l'Europe, avec leur' $300.000 \mathrm{CV}$ en deux centrales.

La Finlande, qui n'a pas de charbon a, elle aussi, compris tout l'intérêt qu'elle pourrait tirer de ses ressources immenses en force hydraulique et elle est en train d'aménager la cascade de Imatia.

L'Autriche, la Russie des Soviets survent le mouvement.

Comme conclusion, et pour en terminer sur cette question, nous donnerons ci-dessous le tableau de la répartition des res sources en force hydraulique des principales puissances euro péennes.

\section{Pays}

Puissance hydraulique

Grande-Bretagne...$\ldots \ldots \ldots \ldots$ possible en $\mathrm{CV}$

Allemagne................... 350.000

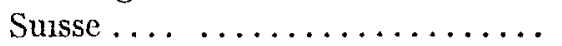

2.050 .000

Italie......................

3.500 .000

France ....................

5.500 .000

Suède... . . ...............

8.000 .000

Norvège...$\ldots \ldots \ldots \ldots \ldots \ldots$

7.000 .000

10.000 .000

Extrait de Science.et Industrie, mars 1929. 